\title{
Research on Localization Algorithm Based on Improved Bayesian Filtering Model
}

\author{
Zeyu Sun ${ }^{1,2}$ and Xiaoguang $\mathrm{Li}^{3}$ \\ ${ }^{1}$ Department of Computer and Information Engineering, Luoyang Institute of \\ Science and Technology, Luoyang 471023, China; \\ ${ }^{2}$ Electrical and information Engineering, Xi'an Jiao tong University Xi'an \\ 710061, China; \\ ${ }^{3}$ Department of Electrical Engineering and Automation, Luoyang Institute of \\ Science and Technology, Luoyang 471023, China \\ lylgszy@163.com
}

\begin{abstract}
Due to the complexity of the algorithm own limitations and environmental parameters, makes for complex work in the process of localization is bigger, computational complexity, a larger error. In order to ensure the reliability and effectiveness of communication data, ensure that improve the localization accuracy, the proposed multi-channel data transmission mechanism, to realize the collection of real-time data information effectively. Based on the traditional localization algorithm principle and error source is analyzed, combined with Bayesian filtering probability model for RSSI localization algorithm was improved, the received signal strength indicator effectively restrain random fluctuations. Through to the node coordinates between areas corrections, make the final balance of signal strength. Experiments show that improved the reliability of the improved algorithm has higher localization accuracy, and shows the validity and the correctness of the algorithm.
\end{abstract}

Keywords: Localization Algorithm, Bayesian filtering model, associated target, sensor nodes

\section{Introduction}

The characteristics of the wireless sensor network can be summarized as two points: have both the functions of the sensor data acquisition and the data transmission function of the network. Any one node, is a wireless sensor, has the perception, the basic function of data acquisition, processing and transmission, the sensor nodes to each other, and the data transfer between sensors to the data acquisition node, usually in the form of wireless transmission. All nodes can be according to the need for mobile. In the process of moving, the whole network topology can accord need and dynamic change [1-2], it can be said that the wireless sensor network (WSN) is a movable, can be to get the data automatically, automatic processing and monitoring data of the network.

WSN is also a kind of particularity of computer network, the mass nodes using the spontaneous form of wireless communication technology into. It has the characteristics of many different from those traditional networks:(1) node is small in size, with limited resources: based on the circuit and micro electromechanical systems evolved from WSN in a day Point of the integration of each part is quite high, so has the advantages of small volume, tend to be restricted with the battery energy, Due to the wide distribution of sensor nodes, the scale is larger, and monitoring of environment is diverse, even the staff Can't reach some of the areas, spread through the air, cannot in a way that is in the battery supply of energy, energy storage For low and not easy to supplement, once the attack 
would have run out of energy and means "death" of the node. Therefore in the WSN Each layer protocol and system architecture design, energy conservation is an important direction. (2) the communication radius is very small, relatively low bandwidth: WSN data transmission is not only is to use a jump can achieve low In the field of energy consumption, so the communication coverage radius generally no more than a few meters. In the network transmission of data information flow Smaller is based on after dealing with the nodes to achieve the effect of. (3) Computing and storage level limited: because WSN application is special, the node's low consumption, low price Features are led directly to the CPU capacity is weak, the results of the storage capacity is small. So, how to use less memory Storage resources and weak capacity to complete more work, is one of the severe challenges faced in the WSN. (4) Node number and adaptive capacity: the number of nodes in WSN, a densely distributed, most has May be more than tens of thousands. Under special circumstances, the wireless sensor networks deployed in relatively remote place, climate Environment is relatively poor, and the maintenance of the node increases the difficulty even unmentionable [3]. So wireless network communication association Discussion must have adaptive ability and reconfigurable characteristics, at the same time, the hardware and software must have a high fault tolerance and strong features. (5) Network dynamics: observer of WSN, - monitoring object and sensor are the three key factors can be moved, and often there is existing node "death" or new nodes involved. The topology of the network changes, the path of the three key factors changes, adjusts ability and reconfigurable characteristics since has been network must have. (6) For data center: a key part of the WSN and sensory data rather than the network hardware. With the data for the whole the core content of the network, you need to form a network at the same time to the perception of data processing and management in the first place, the Internet Technology and database technology to achieve mutual fusion.(7) the center and the organization: all the nodes in WSN has the same features, have distribution which node in advance As the center, all of the nodes by using the method of distributed to balance each other, can be in without interference from organized into a network Winding. No pre-determined center, the network would not have collapsed because of the damage to a node thin, make the entire network of invulnerability Sex and robustness are good.

\section{Formatting your Paper}

At present there are many kinds of WSN localization algorithm of the classification, common method includes the following five categories: Based on the anchor node localization algorithm and the node localization algorithm Based on the pin [4], finegrained (Fine-Grained) localization algorithm and coarse-grained localization algorithm [5], symbol Position and the Physical Location [6], and Concurrent localization algorithm and Incremental localization algorithm [7], Based on distance localization algorithm and Based on the Range localization algorithm [8].(1) based on fault node localization algorithm and the fault node localization algorithm based on the algorithm of anchor node in all nodes in the process of locating, will be able to get their own coordinates relative to the global coordinate system, and based on the algorithm of anchor node relative coordinates, can only be created if needed and keeping them in the same state, a global coordinate system can be finished by way of coordinate transformation. Locating method Based on the output node with DV-Hop, LCB, AHLos and APIT (Approximate Point-Inthe Triangulation Test) etc., rather than on trace nodes are mainly $\mathrm{ABC}$ (Assumptionbased Coordinates) and AFL (Anchor-Free Localization) [9] (2) physical Localization algorithm and symbol Localization algorithm when meet the requirements of the corresponding orientation of symbols can be successfully converted into physical, and vice versa. The difference between the two lies in the symbolic localization for specific applications in reality significantly more strong ability to adapt. In fact most of the 
method can realize the localization of the WSN node physical orientation, in which algorithm is a typical algorithms are Easy Living, the Active Badge, Cricket localization system can be implemented through the corresponding configuration both types of localization. DV-Hop classic localization algorithm for the network as a frame of reference of the requirements of the anchor node proportion is not high, at the same time algorithm has better localization accuracy, the centric algorithm, however, is totally dependent on the network connectivity to achieve node localization performance. Calculation of time and space complexity is low, and easy to implement's node localization algorithm to the network must be considered localization accuracy, extensibility, timeliness, adaptability, computational complexity, robustness and efficiency. There are many algorithms of the localization accuracy, there is certain improvement space, and node localization technology research is still one of the key problems of WSN [10]. (3) the fine-grained localization algorithm and coarse-grained localization algorithm through the received azimuth Angle, time, size and signal intensity pattern matching to locate the information which is known as "fine-grained localization algorithm", typical of Cricket, AHLos, LCB, RADAR and so on; According to the degree of node close distance for localization is coarse-grained localization algorithm, which typically have a convex programming and centric algorithm, etc., (4) incremental localization algorithm and hairstyle is unknown node localization algorithm and hairstyle algorithm by means of parallel to the computation of node location at the same time. To avoid the error accumulation and the degree of local special small is good, and your hair algorithm accounts for most of the proportion, such as ABC. Incremental (Incremental) algorithm is usually 3 or 4 nodes only began to start, and then according to the already completed orientation between the known and unknown nodes of the distance or Angle and other relevant information or simply by using the method of local optimum triangulation gradually approximate solution of unknown node position, the lack of such algorithm is the accumulation of error is larger. Besides localization algorithm there are many other kinds of categories, such as Relative orientation of (Relative) and Absolute (Absolute).Loose affinity (Loosely Coupled) localization algorithm and closely said (Tightly Coupled) localization algorithm, scenario Analysis (Scene Analysis), Triangulation (Triangulation), close (Proximity) localization algorithm [11]. (5) localization algorithm based on distance measuring and localization algorithm [12]; Based on the ranging localization algorithm based on distance measurement is obtained by measuring the information such as the Angle or distance to approximate solution of the actual location coordinates, the algorithm in general can obtain high localization accuracy, but limited in such aspects as hardware conditions and cost easy, in the case of these conditions is restricted location algorithm based on the range of low cost and high efficiency has become the key of the algorithm. Because the latter usually when estimate the distance between nodes or Angle are using the multiple paths of node information exchanging and connectivity to complete position estimate. Now more representative algorithm based on distance measurement are: AHLos (Ad-Hoc Localization System) algorithm [13], Cricket indoor Positioning System, RADAR [14], the APS algorithm based on AOA (Ad Hoc Positioning System) [15], DPE (Directed the Position Estimation) algorithm [16] and LCB algorithm (Localizable Collaborative Body) [17]; Typical localization Algorithm based on the range are: DV-Hop (short Vector-Hop) Algorithm [18], Censored Algorithm (Centric Algorithm) [19], Mobile beacon node (Mobile Anchor Points, MAP) localization Algorithm [20], Convex programming (Convex Optimization) Algorithm [21] and HiRLoc Algorithm [22] and MDS - MAP Algorithm [23]. The typical WSN node localization algorithm based on the distance of the two most representative algorithms is DV-Hop classic localization algorithm and centric localization algorithm. The DV-Hop classic localization algorithm for the network as a reference frame anchor node proportion of request is not high, at the same time algorithm has better localization accuracy, centric algorithm, however, depends entirely on the 
network connectivity to achieve node localization performance. Calculation of time and space complexity is low, and easy to implement's node localization algorithm to the network must be considered localization accuracy, extensibility, timeliness, adaptability, computational complexity, robustness and efficiency. There are many algorithms of the localization accuracy, there is certain improvement space, and node localization technology research is still one of the key problems of WSN.

\section{Problem Defining and Network Model}

\subsection{Problem Description}

The known emission signal strength and the received signal strength are used by RSSI to calculate the consumption of the signal during transmission. The transmission consumption is then transformed into distance by conducting computation to the signal transmission model with theoretical model. In wireless sensor networks, theoretically, spatial location of an unknown node can be determined using dilatation with the RSSI information of four anchor nodes. For the empirical model, before the actual localization, a number of test points are selected. The received signal strength of each base station of these points is recorded to build an off-line database for the relationship between the position of each point and the signal strength. In practical applications, this method has low accuracy because the wireless signal is poorly adaptable to complicated environments. However, the hardware devices used by this method is a few. The strength of the received signal and theoretical or empirical path loss transmission models are used to calculate the distance.

The directed vector set $\mathrm{X}=\left(x_{1}, y_{1}, z_{1}, x_{2}, y_{2}, z_{2}, \cdots, x_{\mathrm{n}}, y_{\mathrm{n}}, z_{\mathrm{n}}\right)$ was used herein to simulate the initial locations of the sensor nodes that were distributed randomly in the network topology area in $\mathrm{R}_{3}$ space. Assume that the locations of $\mathrm{m}$ anchor nodes are unknown, $n-m$ is the number of unknown nodes, and the location of the network is $\left(x_{\mathrm{m}+1}, y m_{+1}, z m_{+1}, \cdots\right.$, $x n, y n, z n)$ Then the localization for network nodes can be described as a process that the coordinate values of the unknown nodes with communication constraints distributed in the network area are solved using the known locations of anchor nodes by geometric theories.

Figure 1 below is called sensor layer, a total of six nodes combination. They collect different types of data, and according to certain rules of a certain number of cluster structure. On Layer called Sinks, its main role is to provide a larger layer of sensor nodes the ability of computing and storage and energy supplement, is responsible for collecting data from sensor layer and go to base station. For the sensor layer, if each node is covered Dry the target node, so formed the associated with the target node attributes. The sensing Between A device node and destination node 1, 2; Sensor node and target node 2 and 3; Sensor nodes between $\mathrm{C}$ and target node 3, 4; D and sensor nodes The target node between June; E sensor node and destination node form between 5 or 6The attribute; When the sensor nodes increases, can be a target node Covered with a sensor node, at this time to form the heavy cover, namely a target node Will appear with more formed between multiple sensor nodes associated attributes. When on a Target node coverage is higher than its own content coverage, can produce a large number of redundant nodes exist, because there are a number of redundant nodes, will inevitably make the whole network Complex system consumes a lot of energy, not for the whole network system to provide longer survival Cycle. As shown in Figure 2. 


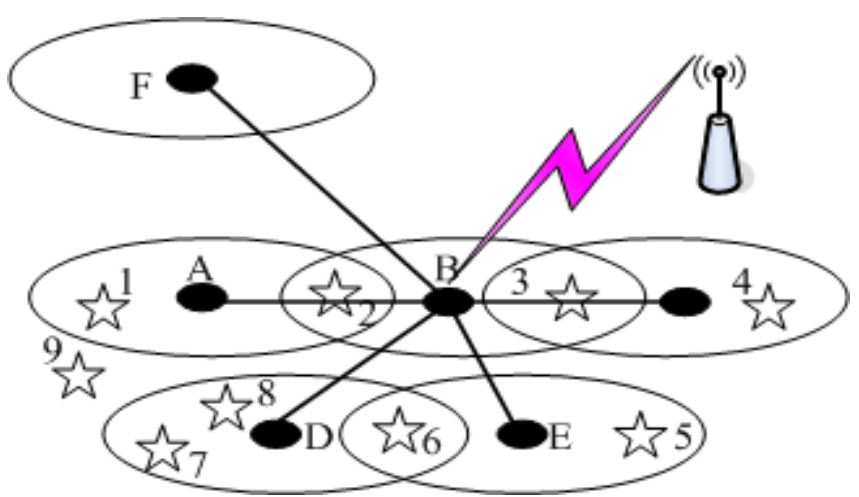

Figure 1. Network Topology Data Fusion

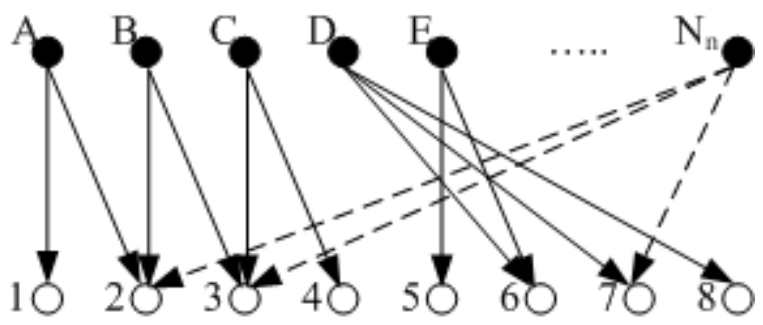

Figure 2. Sensor Node Associated with the Target

In Figure 2, which is formed by the 1 as the foundation of associated with the solid line for sensing in figure 1The link between the node and destination node properties, while the dotted line is when the sensor section. To increase the coverage target node formed by the association. Figure 2, for example, when the Sensor nodes increases, the target node 2, 3,6 in multiple coverage area, in full. The foot under the premise of certain coverage, can produce a large number of redundant nodes. In order to avoid the existence of a large number of redundant nodes, make all sensor nodes in different condition and real now the state transitions between different states.

\subsection{Relation of Distance District Mapping}

The attenuation during sign transmission is used by RSSI to estimate distance. The signal strength keeps decreasing as the transmission distance increases when the signal is transmitting in the space. The distance between the signal sending node and the signal receiving node can be estimated by measuring the strength of the received signal. The mathematical model for signal transmission is

$$
P_{r}(d)=P_{r}\left(d_{0}\right)+10 n \lg \left(d / d_{0}\right)+X_{\sigma}
$$

Where, $P_{r}(d)$ is the received signal strength at the place with a distance of $d$ from the signal sending node, $d_{0}$ is the reference distance; $P_{r}\left(d_{0}\right)$ is the path attenuation coefficient at the reference distance $d_{0}, n$ is the path attenuation index, and $X_{\sigma}$ is the Gaussian distribution random index with the mean value of 0 . In the practical application, the localization accuracy is greatly affected by the path attenuation of wireless relay broadcasting when RSSI signal strength is used to measure distance.

During the localization of wireless sensor networks, if there does not exists any error between the four anchor node and one unknown node, then the distance formula as follows: 


$$
\left\{\begin{array}{l}
\left(x_{1}-x\right)^{2}+\left(y_{1}-y\right)^{2}+\left(z_{1}-z\right)^{2}=d_{1}^{2} \\
\left(x_{2}-x\right)^{2}+\left(y_{2}-y\right)^{2}+\left(z_{2}-z\right)^{2}=d_{2}^{2} \\
\left(x_{3}-x\right)^{2}+\left(y_{3}-y\right)^{2}+\left(z_{3}-z\right)^{2}=d_{3}^{2} \\
\left(x_{4}-x\right)^{2}+\left(y_{4}-y\right)^{2}+\left(z_{4}-z\right)^{2}=d_{4}^{2}
\end{array}\right.
$$

Where ( $\mathrm{x}, \mathrm{y})$ denotes the Euler distance between the coordinate value of unknown node E and the known nodes A, B, C, D. It can be obtained through the Geometry theory and calculation. $\theta_{1}=\operatorname{arctg} \frac{y}{x} \theta_{2}=\operatorname{arctg} \frac{x}{y} \beta_{1}=\operatorname{arctg} \frac{z}{\sqrt{x^{2}+y^{2}}}$ After taking the map point $\mathrm{F}$ as the center of the sphere, and the up section and down section equations are expressed as:

$$
\left\{\begin{array} { l } 
{ y _ { \text { up section } } = k _ { 1 } z } \\
{ y _ { \text { down section } } = k _ { 2 } z }
\end{array} \quad \left\{\begin{array}{l}
k_{1}=\operatorname{tg}\left(\theta_{1}+\theta_{2}\right) \\
k_{2}=\operatorname{tg}\left(\theta_{1}-\theta_{2}\right)
\end{array}\right.\right.
$$

Similarly, the remaining four plane equations obtained to determine the geometry of space planes.

\subsection{Relation of Distance District Mapping}

Receiving node, he thought. One is accessible, signal propagation path is a straight line, without reflection refraction, etc., is put forward formula used to calculate the ideal distance in space and the relationship between the signal intensity:

$$
P_{r}(d)=\frac{P_{t} G_{t} G_{r} \lambda^{2}}{16 \pi^{2} d^{2} L}
$$

Formula, $d$ is the distance between the source and receiver, $P_{t}$ is the strength of the transmitted signal, $G_{t}$ and $G_{r}$, Especially in sender and receiver antenna gain, $L(L>=1)$ to system losses, $\lambda$ for wavelength.

The refinement process essentially is a recursive iterative algorithm. On most occasions, the position distance among nodes and the updated actual distance among nodes under binding conditions will make the new position closer to the actual position of the node. After several recursions, the distance error will be reduced gradually. Finally the refinement algorithm will terminate and the final position will be reported. When the neighboring nodes of an anchor node in a three dimensional space are over four, the inferred linear equation set are over determined. Errors can be balanced using the least square method. Although some redundant nodes have some effects on node localization information, the errors between the distance evaluated by theoretical calculation and the actual distance are still reduced.

Errors are inevitable. Roughly they can be classified into three types. The first type of errors is errors of the position coordinates of the reference nodes selected for node localization. The second type of errors is measurement errors of RSSI distance measurement technology. The third type of errors is caused during the computation. Accordingly, further correction and evaluation are needed for the estimated position coordinates. The correction function is:

$$
\Delta f(x)=\sum e_{i j}\left(l_{i j}-d_{i j}\right) p(i \mid j)
$$

where, $l_{i j}$ is the distance between the node $\mathrm{i}$ and the node $\mathrm{j}$, dij is the RSSI measuring distance between the node $i$ and the node $j, e_{i j}$ is the direction unit vector, $p$ (ijj) is the conditional probability of the node $i$ and the node $j$. Order $E(X)$ and $D(X)$ are the mean value and variance of Gaussian distribution function, respectively. The covariance is: 


$$
\operatorname{Cov}(X)=\frac{1}{n-1} \sum_{i=1}^{n}\left(x-x_{0}\right)\left(x-x_{0}\right)^{T}
$$

Then the $\mathrm{j}^{\text {th }}$ probability density of the posterior probability density is:

$$
\begin{aligned}
E\left(Z_{i j}\right) & =p\left(C_{j} \mid X_{i}\right)=p\left(c_{j}\right) p\left(x_{i} \mid c_{j}\right) / p\left(x_{i}\right) \\
& =p\left(c_{j}\right) p\left(x_{i} \mid c_{j}\right) / \sum_{j=1}^{k} p\left(c_{j}\right) p\left(x_{i} \mid c_{j}\right)
\end{aligned}
$$

The position coordinate of the unknown node can be considered as $P_{\mathrm{i}}=P_{\mathrm{i}}+\triangle f(x)$, where $p_{i}$ is the coordinate figure of the node $i$. Repeat the adjustment process. The adjustment of position coordinate is considered to finish when the correct value of the node position tends to the actual value. The main purpose of iteration convergence is to compare the actual coordinate vector with the estimated coordinate vector of any node $i$. Order that the actual coordinate vector and the estimated coordinate vector of the node $i, j$ are $\left(x_{i}, x_{i}\right),\left(x_{j}\right.$, $\left.x_{j}\right)$ and $\left(\hat{x}_{i}, \hat{y}_{i}\right),\left(\hat{x}_{j}, \hat{y}_{j}\right)$ respectively; the actual distance and the estimated distance between the node $i$ and the node $j$ are:

$$
\left\{\begin{array}{c}
d_{i j}=\left\|x_{i}-x_{j}\right\|=\sqrt{\left(x_{i}-x_{j}\right)^{2}+\left(y_{i}-y_{j}\right)^{2}} \\
\hat{d}_{i j}=\left\|\hat{x}_{i}-\hat{y}_{j}\right\|=\sqrt{\left(\hat{x}_{i}-\hat{x}_{j}\right)^{2}+\left(\hat{y}_{i}-\hat{y}_{j}\right)^{2}} \\
E_{r r}=\sum_{i, j} \frac{\left(d_{i j}-\hat{d}_{i j}\right)^{2}}{d_{i j}} / N
\end{array}\right.
$$

The above $E_{r r}$ is a compromising coefficient of the absolute error square and the relative error square of the average one leap distance among all neighboring nodes. $N$ is the total number of the neighboring nodes [24]. Since the absolute errors are directly affected by the length of the measuring distance, the Errs at different communication radius vary too greatly to be used as a general localization error standard. Generally speaking, the measuring error of the distance between nodes increases as the distance increases and the corresponding node localization error also increases. Thereupon, using iterative method can make the distance between the known node and the unknown node closer to the value calculated theoretically.

\subsection{Improved Algorithm}

RSSI is using in the process of signal transmission attenuation distance estimation. Due to the Signal in the process of space transfer, usually with the increase of transmission distance, signal strength falling. By measuring the received signal strength, and can be fired estimated node the distance between the receiving nodes. Mathematical model for its spread:

$$
P_{r}=P_{r}\left(d_{0}\right)+10 n \lg \left(\frac{d}{d_{0}}\right)+X_{\sigma}
$$

In repeated measurements for many times, and assumes that each measure is independent and obtaining the RSSI measurement is independent distribution. The algorithm of Bayesian probability is to signal strength as normal distribution probability model. In the RSSI, due to the received signal strength will eventually reach equilibrium, the Bayesian probability model statistical algorithms to retain as much as possible. The signal strength distribution information, in order to improve the localization accuracy, the model is: 


$$
P(l \mid o)=\frac{P(o \mid l)}{P(o)}=\frac{P(o \mid l)}{\sum P\left(o \mid l^{\prime}\right) P\left(l^{\prime}\right)}
$$

Among them, $\mathrm{P}(l \mid \mathrm{o})$ is the unknown node $l$ located in corresponding observation signal intensity template the distribution probability of $o ; \mathrm{P}(l)$ is the unknown node position in $l$ prior probability, can be $\mathrm{P}(l)$ hypothesis for a normal distribution model. $L$ is a collection of all the beacon nodes, and $\mathrm{P}(o)$ can be as a standard constant. When the signal strength calculation, are false with average $\sigma$ and standard deviation of the $x_{i}$ Gaussian distribution, the $\sigma$ is the model for adjustable the parameters of the section. The measured results, relative to the $x_{i}$ density function of $\mathrm{x}$.

$$
f(x)=\frac{1}{\sigma \sqrt{2 \pi}} e^{\frac{\left(x-x_{i}\right)^{2}}{2 \sigma^{2}}}
$$

The refinement process is actually a kind of loop iteration algorithm, the node through a series of steps the flash update its position, and written to the database again. In the beginning of each step, node broadcast own position value calculation, and from the neighbor node receives the position information and phase should be the distance of the valuation, and then by triangulation algorithm to solve the least squares method set it to a new location. In most cases, position distance between nodes and is about Beam under the condition of actual distance of the updated node will force more close to the new location the true location of the nodes. When after many times of circulation, the range error progressively small, actuarial method was terminated and reports the final location. When a three-dimensional space anchor node number of neighbors more than 4 , deduces the linear system of equations is over determined, using the least square method can balance error. Although there are some redundant nodes the node location information have influence, but through the theoretical calculation and the actual distance between the errors is on the decrease. A node selected reference node coordinates itself has certain error; Second, the error of the measured RSSI ranging technology; third, in the process of calculation is also produced A certain error. That requires further amendments to the estimated position coordinates and discriminate, fix it is function:

$$
\Delta f(x)=\sum e_{i j}\left(l_{i j}-d_{i j}\right) p(i \mid j)
$$

Among them, $l_{i j}$ is the distance between node $i$ and $j . d_{i j}$ is the RSSI nodes $i$ and $j$ the measured distance; $e_{i j}$ unit vector for direction; $\mathrm{P}(i \mid j)$ is a node $i$ and $j$ A probability. $E(X)$ and $D(X)$ for the mean and variance of the Gaussian distribution function, association The variance:

$$
\operatorname{Cov}(X)=\frac{1}{n-1} \sum\left(x-x_{0}\right)\left(x-x_{0}\right)^{T}
$$

The posterior probability density is the first $j$ a probability density:

$$
E\left(Z_{i j}\right)=P\left(C_{j} \mid X_{i}\right)=\frac{P\left(C_{j}\right) P\left(X_{j} \mid C_{j}\right)}{P\left(X_{i}\right)}=\frac{P\left(C_{j}\right) P\left(X_{j} \mid C_{j}\right)}{\sum P\left(C_{j}\right) P\left(X_{i} \mid C_{j}\right)}
$$

$N$ is the total number of all adjacent nodes, Because of the absolute error varies directly with the length of the distance measurement, in different flux when $E_{r r}$ Radius $r$ change is too big, not as a common localization error criterion [25-26]; Because the distance between the nodes increases generally with the value of distance measurement error, phase Should the node localization error are also increase, so using the iterative solution can make known node and the distance between the unknown node is more close to the theoretical calculation values. 


\section{Estimation System}

In order to verify the experimental process and conclusions, this paper did some experiments on RSSI algorithm. Five kind of coordinate's value were adopted in order to get the convenience for experiment. MATLAB6.5 was applied as the simulation platform and simulation area was $100 \times 100$. Anchor nodes uniformly distributed in the simulation area, and any one of the unknown node can receive the anchor node to periodically send the message, which the coordinates with the localization error in Table 1.

Table 1. List of Coordinates and Localization

\begin{tabular}{llll}
\hline Algorithm & $\begin{array}{l}\text { Actual } \\
\text { coordinates }\end{array}$ & $\begin{array}{l}\text { Calculation of } \\
\text { coordinates }\end{array}$ & $\begin{array}{l}\text { Localization } \\
\text { error }\end{array}$ \\
\hline RSSI algorithm & \multirow{2}{*}{$(19,22)$} & $(19.331,22.219)$ & 0.329 \\
paper algorithm & & $(16.091,22.013)$ & 0.192 \\
RSSI algorithm & \multirow{2}{*}{$(27,35)$} & $(27.823,36.102)$ & 0.823 \\
paper algorithm & & $(27.409,36.061)$ & 0.677 \\
RSSI algorithm & \multirow{2}{*}{$(40,50)$} & $(40.198,50.208)$ & 0.287 \\
paper algorithm & & $(40.142,50.171)$ & 0.222 \\
RSSI algorithm & \multirow{2}{*}{$(68,59)$} & $(68.212,59.277)$ & 0.226 \\
paper algorithm & & $(68.095,59.002)$ & 0.181 \\
RSSI algorithm & \multirow{2}{*}{$(90,87)$} & $(90.285,87.151)$ & 0.260 \\
paper algorithm & & $(90.112,87.008)$ & 0.129 \\
\hline
\end{tabular}

As can be seen in Table 1, the errors of the optimizing value provided in this study were less than those of the value calculated using RSSI algorithm. Moreover, the smaller the distance between the coordinate values of the real node and the coordinate values of the calculated node was, the smaller the error was. Thus, overall the accuracy was improved to a certain extent. When the distance between the coordinate values of the calculated node and the coordinate values of the real node increased, the error would increase accordingly. When the number of the beacon nodes increased, however, the error ratio would decline.

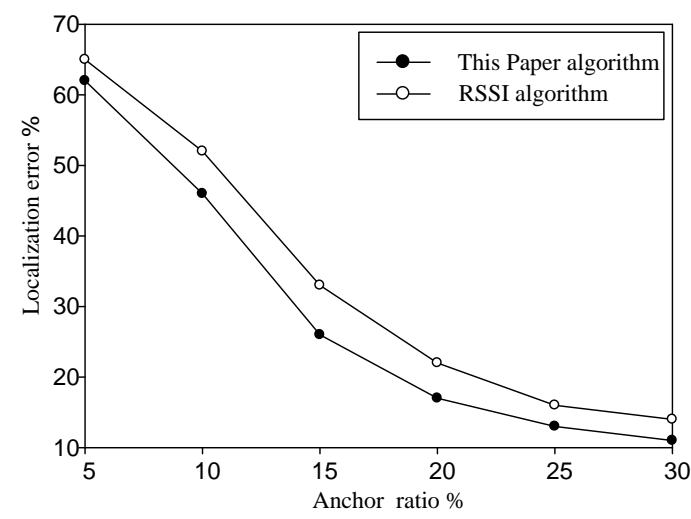

Figure 3. The Relation of Anchor Nodes and Localization Error

Anchor nodes and unknown nodes were evenly distributed in the simulation area. There were 20 anchor nodes, each one with a communication radius of $10 \mathrm{~m}$. The experimental data were from the mean values of 50 simulation tests. Given a determined number of nodes, the localization error would decrease as the dynamic increase of the beacon nodes, as shown in Figure 3.

The network environment in Figure 2 was made up of 200 randomly distributed nodes, and the total number remained consistent. Different ratios of the beacon nodes to the total nodes were taken to do the computation, showing that the localization accuracy of both 
the two algorithms increased as the number of the beacon nodes increased. When the ratio was from 15 to 20 percent, the localization accuracy of the proposed algorithm was obviously higher than that of the RSSI algorithm. When the ratio was close to $25 \%$, the localization accuracy was also close to balance. For the same localization accuracy, it was obtained through iteration refinement calculation for several times by the proposed algorithm while it was obtained by the RSSI algorithm through considerable consumption of time and network power.

A certain number of nodes were randomly distributed in the simulation area, among which the anchor nodes accounted for $20 \%$ of the total number of nodes. MATLAB6.5 was applied as the simulation platform and simulation area was $300 \times 300$. When the total number of nodes increased, the number of data packets used to position increased sharply. Compared with the RSSI, the quantity of the information packets in this study obviously reduced, as shown in Figure 4.

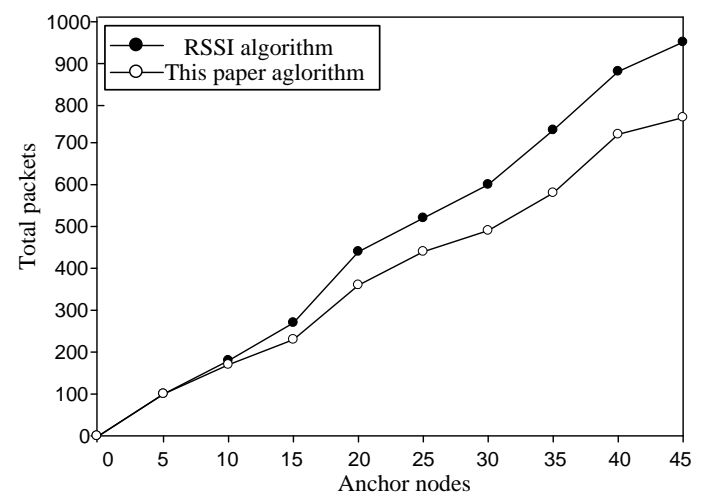

Figure 4. Network Communication Cost

As shown in Figure 4, when the number of the anchor nodes remained consistent, the network communication overhead of the proposed algorithm was less than that of the RSSI algorithm, whereupon the power consumption between nodes was also less than that of RSSI algorithm. In summary, the proposed algorithm reduced the power consumption of the nodes, extended the life cycle of the network, and improved the network stability.

\section{Conclusion}

Node localization was studied in this study based on RSSI and combining with relevant theoretical knowledge of geometry. Simulation tests were conducted to the system model using the distance mapping relation [27]. Through the comparison with RSSI algorithm, it was verified that the proposed algorithm was superior to the RSSI algorithm either in localization error or in packet throughput of network information. The effectiveness of the proposed algorithm was also verified. Because the proposed algorithm is subject to the effect of the accumulative localization errors and communication data of redundant nodes, its application in super size sensor network is to be studied further.

\section{Acknowledgements}

The authors wish to thank Science of Technology Research of Foundation Project of Henan Province Education Department under Grant Nos.2014B520099; Natural Science and Technology Research of Foundation Project of Henan Province Department of Science under Grant Nos. 142102210471, 142102210063; Natural Science and Technology Research of Foundation Project of Luoyang Department of Science under Grant Nos. 1401037A. 


\section{References}

[1] Y. C. Tseng, P. Y. Chen and W. T. Chen, "K-Angle object coverage problem in a wireless sensor network", IEEE Sensors Journal, vol. 12, no. 3408, (2012).

[2] S. Mini, S. K. Udgata and S. L. Sabat, "Sensor deployment and scheduling for target coverage problem in wireless sensor network", IEEE Sensor Journal, vol. 3, no. 636, (2014).

[3] Y. Jin, J. Y. Jo, L. Wang, Y. Kim and X. Z. Yang, "ECCRA: An energy-efficient coverage and connectivity preserving routing algorithm under border effects in wireless sensor networks", Computer Communications, vol. 10, no. 2398, (2008).

[4] S. K. Shekofteh, M. B. Khalkhali and M. H. Yaghmaee, "Localization in wireless sensor networks using tabu search and simulated annealing", 2010 The $2^{\text {th }}$ International Conference on Computer and Automation Engineering, vol. 2, no. 752 (2010).

[5] R. X. Li, Z. Y. Fang and B. C. Hao, "Research on indoor wireless localization system for radioactive sources based on ZigBee", Computing Control and Industrial Engineering, vol. 2, no. 359, (2010).

[6] Z. Yi and C. Koaihei, "Sensor Deployment and target localization in distributed sensor network", ACM Transactions on Embedded Computing Systems, vol. 1, no. 61, (2004).

[7] C. Y. Lin, W. C. Peng and Y. C. Tseng, "Efficient In-Network moving object tracking in wireless sensor network", IEEE Transactions on Mobile Computing, vol. 8, no. 1044, (2006).

[8] H. M. Ammari and S. K. Das, "Centralized and clustered k-coverage protocols for wireless sensor networks", IEEE Transactions on Computers, vol. 6, no. 118, (2012).

[9] K. I. Chakrabarty and H. R. Qi, "Grid coverage for surveillance and target location in distributed sensor network", IEEE Transactions on Computers, vol. 12, no. 1448, (2002).

[10] S. Wang and K. Z. Liu, "A method improving accuracy of self-localization in wireless sensor networks", The $7^{\text {th }}$ World Congress on Intelligent Control and Automation, vol. 1, no. 1748, (2008).

[11] P. Neal and O. Alfred, "Manifold learning algorithm localization in wireless sensor network", International of Conference Acoustics, Speed and Signal Processing, vol. 3, no. 857, (2004).

[12] D. Niculescu and B. Nath, "DV-based positioning in ad-Hoc network", Journal of Telecommunication Systems, vol. 4, no. 267, (2003).

[13] O. Songhwai, S. Sastry and L. Schenato, "A Hierarchical multiple target tracking algorithm for sensor network", Proceeding of the 2005 IEEE International Conference on Robotics and Automation, vol. 4, no. 2197, (2005).

[14] A. Arora and M. Kansiels, "A line in the sand: a wireless sensor network for target detection, classification, and tracking", Computer Network, vol. 5, no. 605, (2004).

[15] H. Qi, Y. Xu and X. Wang, "Mobile-agent-based collaborative signal and information processing in sensor networks", Proceeding of the IEEE, vol. 8, no. 1172, (2003).

[16] S. L. Zhou, Y. Xiao and S. C. Wu, "Target tracking system based on wireless sensor network", 2010 International Conference on Digital Manufacturing and Automation, vol. 2, no. 599, (2006).

[17] M. Cardei and D. Z. Du, "Improving wireless sensor network lifetime through power aware organization", Wireless Networks, vol. 3, no. 333, (2005).

[18] F. Zhao, J. Shin and J. Reich, "Information driven dynamic sensor collaboration for tracking application", IEEE Signal Processing Magazine, vol. 3, no. 61, (2002).

[19] M. Mechitov, S. Sundresh and Y. Kwon, "Cooperative tracking with binary detection sensor network", Proceeding of the $1^{\text {th }}$ International Conference on Embedded Networked Sensor Systems, vol. 1, no. 332, (2003).

[20] S. Pattem, S. Poduri and B. Krishnamachari, "Energy quality tradeoffs for target tracking in wireless sensor network", Proceeding of the $2^{\text {th }}$ Workshop on Information Processing in Sensor Networks, vol. 1, no. 32, (2003).

[21] D. Tian and N. D. Georgansa, "Anode scheduling scheme for energy conservation in large wireless sensor network", Wireless Communications and Mobile Computing, vol. 3, no. 271, (2003).

[22] H. Liu, P. Wan, C. W. Yi, X. H. Jia and P. Niki, "Maximal lifetime scheduling in sensor surveillance networks", IEEE Transactions on Computers, vol. 11, no. 557, (2011).

[23] K. F. Su, W. H. Ousiai and H. C. Jiau, "Localization with mobile anchor points in wireless sensor networks", IEEE Transaction on Vehicular Technology, vol. 3, no. 1187, (2005).

[24] S. Zeyu, W. Weiguo and W. Huanzhao, "A Novel Coverage Algorithm Based on Event-ProbabilityDriven Mechanism in Wireless Sensor Network", Journal on Wireless Communications and Networking, vol. 4, no. 1, (2014).

[25] Y. X. Xu, X. Gao and Z. Y. Sun, "WSN node localization algorithm design based on RSSI Technology", 2012 Fifth International Conference on Intelligent Computation Technology and Automation, vol. 1, no. $556,(\mathbf{2 0 1 2})$.

[26] S. Zeyu, W. Weiguo and W. Huanzhao, "An Optimized Strategy Coverage Control Algorithm for WSN", International Journal of Distributed Sensor Networks, vol. 6, no. 1, (2014).

[27] S. Mini, S. K. Udgata and S. L. Sabat, "Sensor deployment and scheduling for target coverage problem in wireless sensor network", IEEE Sensor Journal, vol. 3, no. 636, (2014). 


\section{Authors}

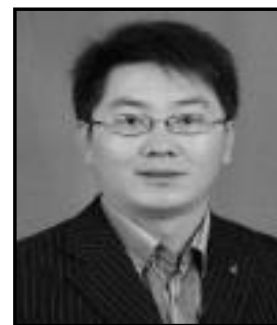

Zeyu SUN was born in 1977 in Changchun city, Jilin province, in 2010 graduated from Lanzhou University, Master of Science; Xi an Jiao tong university study for $\mathrm{PhD}$ at present. $\mathrm{He}$ is a lecturer in Luoyang institute of technology of computer and information engineering, is also a member of China computer society. The main research interest is in wireless sensor networks, parallel computing and Internet of things.

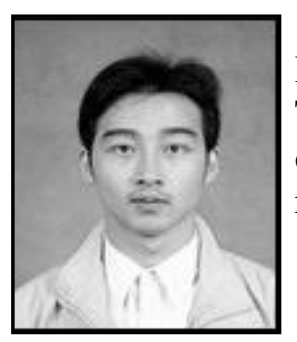

Xiaoguang LI was born in 1983 in Zhoukou city Henan province. In 2010 graduated from Huazhong University of Science \& Technology, Master of Science; He is a lecturer in Luoyang institute of technology of Electrical Engineering and Automation. The main research interest is industry automation, image processing. 